\title{
Study on Teaching Strategies in Mathematics Education based on CAI
}

\author{
Yan Feng Wei \\ Teacher's College, Beihua University, Jilin City, China
}

\begin{abstract}
With the development of information technology and the popularization of internet, mobile phone, new media represented is gradually influencing and changing people's study and life, become the centre and social consensus of cultural information, according to the China Internet Network Information centre, the youth is the main use of CAI(Computer Assisted Instruction), which is the most active group of customers, fully understand the impact of the new media environment for students, higher mathematics education of college students in CAI. In this paper, the $\mathrm{CAI}$ is proposed for mathematics education of college students.
\end{abstract}

\section{General instruction}

according to "Report of China Internet Development Statistics" in July 2013 by the China Internet Network Information Centre released 32nd (CNNIC) data show that "As of the end of June 2013, the scale of China reached 591 million Internet users, Internet penetration rate of $44.1 \%$, where 20 -year-old to 29 -year-olds accounted for $29.5 \%$ of all Internet users among the largest proportion of high school students, to 26.8 percent." Internet, mobile phones and other new media appear to accelerate the rhythm of people's lives, so that students become very con-fused and impetuous; in the new media environment presents information uneven, Western countries control the discourse of new media, these phenomena to the development of higher mathematics education has brought many new problems, while the higher mathematics education of college workers is also a great challenge.

With the development of information technology to the Internet, mobile phones represented by the new media has gradually come into our lives, as our access to information, interpersonal communication and entertainment were an indispensable tool in different disciplines and researchers are beginning to focus on this one issue, the higher mathematics education is an important social practices, should continue to meet the development needs of modern society.

How to carry out higher mathematics education in the new media environment is the higher mathematics reality we must face university educators and historic mission. The term "new media" comes from the West, the Western countries to study new media has 40 years of history, our focus on new media is only in recent years things, mostly general, introductory study, not yet formed complete theoretical system of systematic, so contemporary to the higher mathematics education in the context of new media continue research in order under the guidance of Marxism, and in a correct understanding of the basis of new media theory, combined with the actual situation of China's college students proposed countermeasures for our own education.

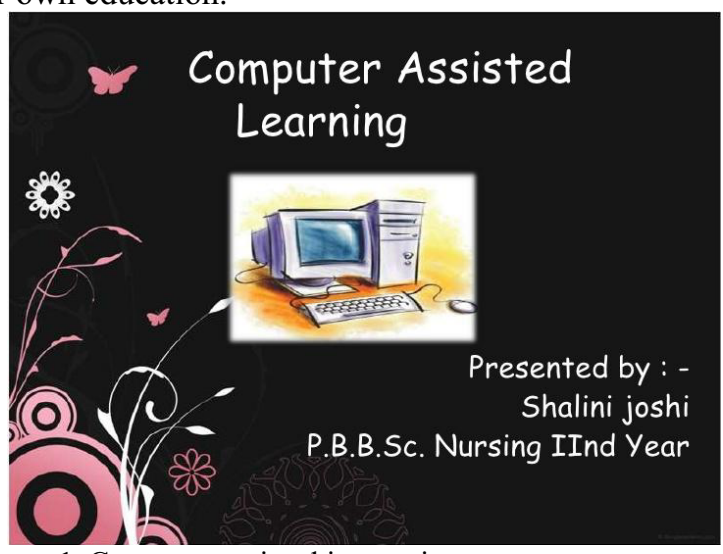

Figure 1. Computer assisted instruction

\section{The traditional higher mathematics education}

New media to expand the dissemination of spatial information, people can at any time, anywhere access to information through new media, and according to their needs and preferences, to form their own independent media space, while traditional media often do not have this feature. Traditional media are unidirectional linear propagation, is a little on the multi-faceted communication, new media, communication style are spread, you can achieve "many to many" communication, is spread by everyone, everyone can be among the audience and interchange the $\mathrm{AC}$ content. The traditional news media no longer dominate the mathematics', more and more of the audience began to mathematics in the 
dissemination of information, and even become a source of information and the publisher, the mainstream media no longer grasp the absolute right to speak, many of whom are involved in college students. The emergence of new media has changed the past, people tuning in via radio and television programs must be synchronized features, at any time to obtain information the audience can select according to their own circumstances, if you are interested you can repeatedly watch the same programs.

The development of digital technology, the economy, politics, culture and society have had a profound impact on the application and research of new media mathematics to get people's attention, especially in recent years, with the development of $3 \mathrm{G}$ technology, some scholars have begun to attention to the relationship between the new media environment and the higher mathematics education among college students, recognizing the rapid development and application of new media to bring new development opportunities to the higher mathematics education in colleges and universities, research new media applications in the higher mathematics education activities, and made some useful results.

2009 Ministry of Education, Social Science Centre hosted "in the new media technology background of higher mathematics education of college students' seminars, conferences, studies the" contemporary college students use new media tools of the status quo and development trend of ideological and new media environment, learning and life the impact of new media to enhance the method by means of higher mathematics education. "the meeting noted that the new media technology platform to broaden the higher mathematics education, higher mathematics means to enrich education, provide a new perspective for the traditional higher mathematics education workers, is conducive to enhancing the practical effect of the higher mathematics education, but also should be noted that under the new media environment impact of a flood of information on college students, is apt to cause physical and psychological double crisis college students, college is not conducive to the formation of a correct world outlook, outlook on life and values. Higher mathematics Education Workers in the actual teaching work to change the educational ideas to improve the understanding of new media, and actively explore ways and laws of the higher mathematics education of college students in the new media environment, strengthen the construction of university networks, active occupation Ideological new Media positions of political education.

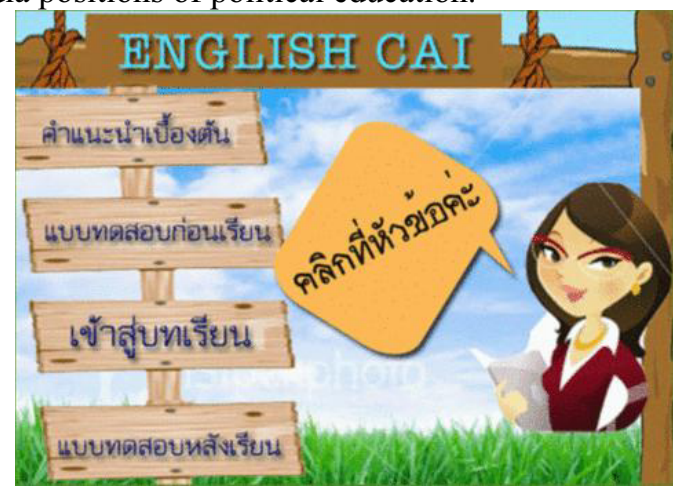

Figure 2. Computer Assisted Instruction scene renderings

\section{Computer multimedia technologies in mathematics foundation course}

Computer multimedia technology in mathematics foundation course in computer applications in mathematics teaching universities are generally placed in specialized courses designed mathematics lesson, rarely involves basic course, most teachers believe that the traditional mathematics of teaching is the key to solving the shape of basic skills students. But the study, we found that the traditional teaching of basic courses in computer multimedia teaching should be assisted. In this regard, we can learn on the computer and briefly studied mathematics analysis. We know that the traditional mathematics of teaching is mainly on the basis of sketches and colour teaching, teaching in the sketch is mainly to solve our modelling capabilities, modelling training, perspective, anatomy, sense of space, texture, composition and other content; colour teaching in our main solution hue contrast, colour contrast, complementary colour contrast and colour space and colour contrast and well-being and so on. We discuss these issues now can be done in a number of software, ie for colour sketch studies can be carried out entirely in the computer. Such teaching and research can save a lot of time, avoiding the production of hand-painted dull duplication of effort. Take a colour mosaic effect work constitutes an example, if you want to complete a form, you need to repeatedly create and modify, wasting a lot of time and effort. The application of computers to complete simply enters an image in Photoshop software, and then performs Filter- Pixy elate- Mosaic command, a mosaic work done in an instant.

We also found that there are some modern software can simulate the effect of a variety of drawing tools, has now developed software such as Fractal Design Painter is good painting software. It provides a lot of painting tools, such as pens, pencils, brushes, watercolour pen, oil paints, airbrush, crayons, etc., but also offers a wide variety of strokes, to a certain extent, truly mimics the effect of freehand drawing. Such as when using painting tools, mimic Van Gogh, Cezanne and other masters of the brush strokes; when the brush tool, combined with wet drawing paper, the effect of Mathematics on rice paper for the imitable. Although it can not fully replace the painting, the painting can be used as a research method and means. In this way, students can save a lot of freehand drawing practice time, students can also develop visual thinking and analytical skills, help cultivate innovation ability.

Computer multimedia technology in mathematics appreciation class application is an important mathematics' of mathematics class mathematics teaching content. The purpose of mathematics appreciation, mainly in fine mathematics s teaching in two ways: first, through the appreciation of works of mathematics, to understand the history of mathematics development, grasp the law of development of fine mathematics s; second, through appreciation, inspire students' creative thinking, and thus create outstanding works. Through 
appreciation teaching courses on pictures or slides are operations due to the complexity of the fuzzy, often not very efficient. Using computer multimedia teaching, mathematics appreciation class effect is greatly changed. As long as teachers have a multimedia computer, there is mathematics appreciation information and data to a disc loaded into the optical drive (CDROM), students in the appreciation of classical works, a little mouse, a painter's masterpiece and biographies will appear, not only can get a glimpse of picture works, you can also zoom in mathematics observation; not only a comprehensive view of the works, you can also choose to enjoy or have a work print.

Students in the classroom can break time, space, geographical restrictions, and foreign gallop across the ancient and modern, mathematics gallery stroll. Appreciate the mathematics of architecture, when not only the usual way to appreciate the buildings form of visual perception in the stationary state, but also can do dynamic display, allowing the building to spin up, enjoy the multiple sides of the building. You can also incomplete, or damaged historic buildings were restored. Enjoy a graphic design or cover design time, but also can be altered according to their preferences, but it is also indispensable for students to creative material. These are the traditional appreciation class unmatched.

Computer multimedia technology in computer is professional courses in mathematics, as a modern tool, first used in mathematics class in design teaching. It is widely used in the design, liberated the previous complex design work, improve work efficiency, the designer's right-hand man.

The accuracy is concerned, with the computer to produce an object the size of an absolute right, almost no errors. Such as the use of CAD software or 3DMAX produced graphics, with an accuracy of less than $0.1 \mathrm{~mm}$, it can show the most delicate mathematics' of the design, which is the traditional hand-painted difficult to achieve, but its camera angles, pleasant to the eye point of view, depending on from fully simulate the real, not the existence of handmade randomness and imprecision. Therefore, the authenticity of computer design by the majority of designers agrees. In recent years, the development of software used in graphic design software is gradually increasing, a common two-dimensional CorelDraw, Photoshop, AutoCAD, and three-dimensional 3DMAX other software.

In painting courses teaching, there must be a lot of painting techniques require students to master, every teacher of painting styles, techniques have their own characteristics. The instructor is necessary to allow students to expand their horizons, to absorbing. Application of multimedia technology, breaking the teachers' knowledge of this one-way transmission of information sources, expanding the scope of information exchange teaching, breaks the teaching space, so that unrestricted access to information and delivery time and space. For example the Central Academy of Fine Mathematics s masters editing techniques textbooks, covering drawing, painting, oil painting techniques, such as content, teachers can combine curriculum content for teaching demonstration, in the classroom so that students can see the famous painter's lecture demonstrations, but the key place to repeat Play, learn to understand the true meaning of painting masters. Similarly, professional instructors techniques can also be made from their own multimedia courseware demonstration teaching, improve teaching effectiveness.

\section{Mathematics educations is basically synchronized}

Mathematics as a symbol of Chinese mathematics, has developed a number of years, has experienced a long history. Its development and development of traditional mathematics education is basically synchronized. Traditional mathematics education model for studying the development of self-contained mathematics provides a strong guarantee, but the crisis has brought to the development of Mathematics, Mathematics may lead to the development of stagnation and rigid.

With the introduction of Western culture, contemporary mathematics education has been given a new content and features, traditional mathematics education has been seriously challenged, and Mathematics is no exception. Chinese and Western cultures fierce collision, given the contemporary mathematics education conform to the trend of the times, and the Chinese have a tendency to weaken the function of painting, we can see the impact on Mathematics contemporary mathematics education development is enormous. Therefore, Mathematics to have sustainable development, we must look for a path.

Given the cultural heritage and cultural traditions there are significant differences among different ethnic, fusion of contemporary mathematics education and the presence of significant challenges, mainly as follows: Western contemporary mathematics education curriculum is based on the theory, although trying to achieve Western reconcile, western system in order to mark the traditional mathematics education is based on the poetry of learning-based, it is trying to achieve a comprehensive innovation in the original basis. Since the mathematic sing point of contemporary mathematics education and traditional mathematics education, the focus is different. Should contrary to objective laws, not according to the actual situation of contemporary mathematics education and the development of Mathematics, blindly impose two very different systems together, will generate a lot of negative impact, but we should make an objective assessment, can not ignore the contemporary the positive role of mathematics education for the development of Mathematics.

Contradictions are opposites, we study the impact of Chinese contemporary mathematics education in the process of painting effects, specifically requires an objective analysis of contemporary mathematics education teaching, writing produced. This mathematics from the aspect of two dimensions, the use of comparative approach, in teaching, through the teaching objectives, teaching methods, teaching materials, teaching content, teaching evaluation analysis of 
differences in traditional and contemporary mathematics education mathematics education; in the creative aspects of , will be an inspiration on Mathematics, technique, emotion comparative study, summed up the pros and cons of contemporary mathematics education for the development of mathematics, but overall, pros and cons, more harm than good.

Because of our long-term in a feudal society, and the ancient social, political, cultural and systems are designed to meet the needs of the ruling class. The prevalence of the traditional hierarchy is mathematics education, just to satisfy the interests of the ruling class and the service. In this case, the role of mathematics education is major with a strong "into enlightenment, helping Fallon," the. In this historical context, the traditional mathematics education does not have a complete and standardized education system. In mathematics oculars the teaching process, the main idea is to master personal education based, then painting learners main objective things through observation and copying works of the classical way to achieve the purpose of drawing creation.

Visible, education has become the most basic rights of every person, and not a few aristocratic privileges, which will benefit the construction of a socialist harmonious society. Contemporary mathematics education teaching objectives conducive to the development of our comprehensive quality education, improve the overall level of China's national mathematics knowledge. For the construction of a beautiful home that has everything to gain but no harm. However, we also see the shortcomings can not be ignored him an objective reality. Universality corresponds to the peculiarities of contemporary mathematics education is universal knowledge for all students in the mathematics of Mathematics teaching goal setting, and not as a traditional mathematics education that, according to each student's specific conditions, can amount tailor the most appropriate teaching painting learning objectives and teaching goals at any time to adjust according to the needs of learning. Contemporary Mathematics by differences in education and Mathematics teaching objectives analysis, summed up contemporary mathematics education exists on the pros and cons of teaching objectives. We can see that they are closely related, the latter is dependent on the former, while drawing on Western mathematics education.

\section{Conclusions}

Computer mathematics multimedia technology in teaching, not only to speed up the progress of the mathematics of teaching, improve learning outcomes, more important is that it can give students a more novel stimuli, resulting in the best area of the cerebral cortex related development, so as to stimulate their creative evolving thinking. To innovative works are mathematics majors. The information age, computer multimedia teaching the mathematics of teaching is the direction of development, however, stressed the role of modern teaching media is not to deny the traditional teaching, but rather calls attention to a combination of both in teaching practice, flexible use. Contemporary mathematics teaching focus on the integrated is use of multimedia in order to continuously improve the quality and efficiency of the mathematics of teaching. The main trends in the mathematics of teaching is the traditional teaching media development and integration of modern teaching media, and gradually form a complete, current information society to adapt to the development of mathematics education system.

The concept of new media technology director of the Institute was first proposed by Mark P. Gold by the American television network CBS (CBS) in 1967, the concept of new media, academia is also formed without a fully unified conclusion, current consensus view is that new media is relying on network technology and digital technology, the Internet, mobile phones and digital television media representatives, to the public to provide personalized information and entertainment services spread form.

\section{References}

1. Sun Zhuanbo, Guan Hui. Decided to investigate the factors basketball shooting overcast. Harbin Institute of Physical Education, 26(2006)

2. Yuan Ting Gang. A comparative study of two EMG muscle strength training methods and the characteristic parameters. State Sports General Administration of Sports Science Research Thesis. Beijing: State Sports General Administration of Sports Science Institute, 155(2000)

3. Xu honest, Bing-Hong, Wen-Hai Liu. And so on. A comparative study of vibration when the vibration strength training with non-EMG changes. Xi'an Institute of Technology, 54(2004)

4. YANG Haiping. Basketball players of different positions and excellent pitcher perception characteristics. Guangzhou Physical Education Institute. 52(2005)

5. Lu Aiyun. Biomechanical characteristics under different weight men snatch action. Shanghai Institute of Physical Education, 61(2000)

6. Liu Yajun. EMG Tennis basic technology research. Tianjin Institute of Physical Education. 18(2005)

7. Cao Hui. Speed skating leg muscle coordination synchronized EMG analysis grieved. Tsinghua University. 45 (2005) 